\title{
Perfil dos Grupos de Pesquisa em Neurofisiologia do Brasil
}

\author{
Profile of Neurophysiology Research Groups of Brazil \\ Aline dos Santos Vieira ${ }^{1}$, Marcos Roberto Torres Welter ${ }^{2}$, \\ Pâmela Billig Mello-Carpes ${ }^{3}$
}

\section{RESUMO}

Objetivo. Determinar o perfil dos Grupos de Pesquisa (GP) em Neurofisiologia no Brasil, registrados no Diretório dos Grupos de Pesquisa (DGP) do Conselho Nacional de Desenvolvimento Científico e Tecnológico $(\mathrm{CNPq})$, verificando a distribuição geográfica e institucional, bem como perfil de composição dos membros. Método. Foram utilizados dados públicos obtidos junto ao DGP e nos currículos da Plataforma Lattes dos líderes. A pesquisa utilizou a palavra-chave "Neurofisiologia" e foi efetuada entre janeiro e abril de 2013. Resultados. Foram encontrados $52 \mathrm{GP}$ com ao menos uma linha de pesquisa relacionada à Neurofisiologia. $71,15 \%$ dos grupos são de instituiçôes federais, $17,31 \%$ de particulares e $11,54 \%$ de estaduais. $\mathrm{O}$ número total de membros é de 1326; 35\% são pesquisadores, $61,46 \%$ estudantes e 3,54\% corpo técnico. Quase metade dos GP foi criada nos últimos 5 anos, sendo que a maior parte atua na região Sudeste. Todos os líderes possuem doutorado e a maioria pós-doutorado; a sua produção bibliográfica e orientaçóes concluídas apresentam prevalência na região Sul, seguida da região Sudeste. Conclusáo. A pesquisa continua sendo fomentada prioritariamente em instituiçóes federais e nas regiōes Sudeste e Sul do país, provavelmente devido ao desenvolvimento econômico e educacional das mesmas, além da presença de importantes órgãos de fomento.

Unitermos. Fisiologia, Neurociências, Educação, Sistema Nervoso.

Citaçáo. Vieira AS, Welter MRT, Mello-Carpes PB. Perfil dos Grupos de Pesquisa em Neurofisiologia do Brasil.

\begin{abstract}
Objective. Determine the profile of Neurophysiology Research Groups (RG) of Brazil, registered in the Diretório dos Grupos de Pesquisa (DGP) of Conselho Nacional de Desenvolvimento Cientifico e Tecnológico $(\mathrm{CNPq})$, checking the geographic and institutional distribution, as well as the of the members composition profile. Method. We used public data obtained with the DGP and in the curricula of the leaders in the Plataforma Lattes. "Neurophysiology" was used as keyword and the research carried out between January and April 2013. Results. We found $52 \mathrm{RG}$ with at least one line of research related to Neurophysiology. $71.15 \%$ of groups are of federal, $17.31 \%$ private and $11.54 \%$ statewide institutions. The total number of members is 1326; $35 \%$ are researchers, $61.46 \%$ students and $3.54 \%$ technical staff. Almost half of the RG was created in the last 5 years, being that most acts in the Southeast. All the leaders have doctorate and most postdoc; their bibliographical production and completed guidelines are prevalent in the Southern region, followed by South-East region. Conclusion. Research remains priority fostered in federal institutions and in the Southeast and South regions, due to economic and educational development, and the presence of important founding agencies.
\end{abstract}

Keywords. Physiology, Neuroscience, Education, Nervous System.

Citation. Vieira AS, Welter MRT, Mello-Carpes PB. Profile of Neurophysiology Research Groups of Brazil.
Trabalho realizado na Universidade Federal do Pampa, Uruguaiana-RS, Brasil.

1.Acadêmica de Farmácia, Universidade Federal do Pampa (UNIPAMPA), campus Uruguaiana-RS, Brasil, Membro do Grupo de Pesquisa em Fisiologia (GPFis/UNIPAMPA), Uruguaiana-RS, Brasil.

2.Acadêmico de Fisioterapia, Universidade Federal do Pampa (UNIPAMPA), campus Uruguaiana-RS, Brasil, Membro do GPFis/UNIPAMPA, Uruguaiana-RS, Brasil.

3.Fisioterapeuta, Mestre e Doutora em Ciências Biológicas: Fisiologia, Professora adjunta da Universidade Federal do Pampa (UNIPAMPA), campus Uruguaiana-RS, Brasil, Líder do GPFis/UNIPAMPA, Uruguaiana-RS, Brasil.
Endereço para correspondência: Pâmela Billig Mello-Carpes Laboratório de Estresse, Memória e Comportamento, BR 472, Km 592. Cx postal 118. CEP 97500-970, Uruguaiana-RS, Brasil. E-mail: pamelacarpes@unipampa.edu.br 


\section{INTRODUÇÃO}

Durante a evolução filogenética, o Sistema Nervoso Central (SNC), e em especial as estruturas encefálicas relacionadas com o comportamento e com as funçóes cognitivas e emocionais, se desenvolveram de forma complexa, caracterizando o ser humano e diferenciando-o de seus ancestrais ${ }^{1}$. Desde os seres vivos mais primitivos, a principal função do Sistema Nervoso é propiciar a adaptação ao meio ambiente ${ }^{2}$, assim, a evolução humana fez do seu órgão central, o cérebro, uma peça chave para a sobrevivência da espécie ${ }^{3}$.

Neste contexto, a busca pela compreensão do funcionamento do cérebro e do sistema nervoso é um desafio constante para os cientistas da área de Neurociência e Neurofisiologia. O século XX foi notável para o estudo do cérebro e veio revolucionar o modo como hoje é possível entender as funções (e disfunçôes) do Sistema Nervoso ${ }^{4}$.

A história da fisiologia do Sistema Nervoso ${ }^{5}$, a chamada Neurofisiologia, em qualquer país confunde-se com a história da própria Fisiologia, já que toda e qualquer função do organismo animal, dos invertebrados à espécie vertebrada mais evoluída, o Homo sapiens, é gerada, regulada ou pelo menos modulada pelo Sistema Nervoso5.

A Neurofisiologia é um ramo da fisiologia que tem por objeto de estudo os mecanismos de funcionamento do Sistema Nervoso 5 , como já citado, e interage com as neurociências, uma vez que, utilizando o seu conhecimento, pode-se diagnosticar, tratar e monitorar as funções durante procedimentos específicos que envolvam o Sistema Nervoso. Dentro da Neurofisiologia encontra-se a Neurofisiologia Clínica, que, na sua dimensão funcional, utiliza-se de conhecimentos anatômicos, fisiológicos e clínicos, com finalidades diagnóstica, terapêutica e de monitoração de funções durante procedimentos específicos para a avaliação do Sistema Nervoso Central, Periférico e Muscular ${ }^{6}$.

Sabe-se que a produção científica de um país está claramente associada ao seu desenvolvimento social e econômico. Atualmente o Brasil tem sido reconhecido internacionalmente por estar apresentando um avanço significativo em áreas sofisticadas do conhecimento, antes atributo exclusivo de países desenvolvidos. Este fato deve-se ao importante investimento de instituiçóes de fomento à pesquisa, com considerável melhora da infra- estrutura compatível com a pesquisa científica no país, em particular no caso paulista devido, principalmente, ao apoio da Fundação de Apoio à Pesquisa do Estado de São Paulo (Fapesp) ${ }^{7}$.

$\mathrm{O}$ avanço científico de uma determinada área da ciência depende de inúmeros fatores ${ }^{8}$, como a viabilização de políticas e prioridades em pesquisas que fortaleçam as linhas de investigação a partir das demandas atribuídas para as diferentes áreas de um país ${ }^{9}$. Assim, a partir das orientaçóes do Conselho Nacional de Desenvolvimento Científico e Tecnológico (CNPq) foram concebidos os Grupos de Pesquisa (GP), com o intuito de impulsionar o processo de desenvolvimento científico, possibilitando o compartilhamento de instalaçôes e equipamentos, além de viabilizar a produção e as discussóes organizadas de linhas comuns de pesquisa ${ }^{10}$.

Ainda, os GP foram criados para atender as demandas dos programas de pós-graduação, mestrado e doutorado, que precisavam de espaços para discutir os projetos de pesquisa e seus resultados entre professores, estudantes de graduação e pós-graduação e pessoal de apoio técnico ${ }^{9}$, e têm sido um dos parâmetros utilizados para analisar o desenvolvimento de uma área do conhecimento $^{11}$. Entretanto, na área da neurofisiologia, poucas são as publicaçôes que nos trazem informação de abrangência nacional acerca das atividades científicas desenvolvidas nas instituições de ensino e pesquisa do país ${ }^{12}$.

Dessa forma, e considerando a importância da pesquisa científica nesta área, buscou-se através deste estudo, analisar o perfil dos grupos de pesquisa em Neurofisiologia registrados no Diretório dos Grupos de Pesquisa (DGP) do CNPq do Brasil, verificando a distribuição geográfica e institucional e a composição dos grupos, bem como acrescentar conhecimentos sobre as linhas de pesquisa e os pesquisadores dos GP em Neurofisiologia no Brasil.

\section{MÉTODO}

O mapeamento dos grupos de pesquisa em neurofisiologia no Brasil foi realizado a partir do levantamento de dados obtidos junto ao DGP e nos currículos da Plataforma Lattes de seus membros, ambas bases de dados do CNPq. A pesquisa foi efetuada entre janeiro e abril de 2013, utilizando-se o termo "Neurofisiologia" como palavra-chave. 
O DGP no Brasil constitui um banco de dados que contêm informaçóes sobre os grupos de pesquisa em atividade no país e contém uma base corrente, uma vez que estas informações são atualizadas continuamente pelos líderes de grupos, pesquisadores, estudantes e dirigentes de pesquisa das instituições participantes, e o CNPq realiza censos bianuais, que como são fotografias dessa base de dados ${ }^{13}$.

Foram incluídos na análise dos dados somente grupos certificados pela instituição de origem, conforme informaçôes disponíveis na base de dados. A página de cada um dos grupos identificados foi acessada para coleta dos dados.

Este trabalho consistiu de um estudo descritivo e seu levantamento de dados foi dividido em duas etapas. A primeira etapa analisou os seguintes itens: nome do grupo, formação dos líderes, número e nível de formação dos integrantes, instituições a que pertenciam, bem como se as mesmas eram federais, particulares ou estaduais, a região geográfica e as linhas de pesquisa do grupo.

A segunda etapa da pesquisa, relacionada aos participantes dos grupos de pesquisa, foi realizada pela consulta individualizada do currículo de cada líder junto à Plataforma Lattes do CNPq, na qual foram considerados dados relacionados à formação e titulação, número de pu- blicações e orientações concluídas.

$\mathrm{Na}$ avaliação dos currículos foi adotado como critério de exclusão currículos atualizados em data anterior a 2010, da mesma forma como foi feito para as informações retiradas do Diretório dos GP.

O presente estudo trata-se de uma pesquisa documental na qual foram utilizadas apenas fontes de domínio público, dessa forma não necessitou de aprovação do Comitê de Ética em Pesquisa para o seu desenvolvimento. Todavia, os cuidados éticos na coleta, análise e interpretação dos resultados de forma anônima foram seguidos rigorosamente.

Os dados foram organizados, analisados e apresentados na forma de média \pm desvio padrão e/ou percentuais e as análises foram realizadas através do programa GraphPad Prism ${ }^{\bullet}$ versão 5.0 para Windows (GraphPad Software, San Diego, CA, USA).

\section{RESULTADOS}

Foram encontrados no banco de dados do $\mathrm{CNPq}$ 52 grupos de pesquisa nos quais, ao menos uma linha de pesquisa envolve a neurofisiologia. A Tabela 1 apresenta as principais linhas de pesquisa encontradas e sua distribuição geográfica.

Tabela 1

Principais Linhas de Pesquisa relacionadas à Neurofisiologia e sua Distribuição Geográfica

\begin{tabular}{|c|c|c|c|c|c|}
\hline \multirow[t]{2}{*}{ Linhas de Pesquisa } & \multicolumn{5}{|c|}{ Distibuiçấo Geográfica } \\
\hline & $\mathrm{N}^{1}$ & $\mathrm{Ndt}^{2}$ & $\mathrm{CO}^{3}$ & $\mathrm{Sdt}^{4}$ & $S^{5}$ \\
\hline Neurofisiologia da memória, cogniçáo e expressão comportamental & & 2 & & 1 & 5 \\
\hline Neurofisiologia clínica & & 1 & & 1 & 3 \\
\hline Neurofisiologia do envelhecimento & & 1 & & & 2 \\
\hline Neuroanatomia e neurofisiologia de sistemas sensoriais & 1 & & & & \\
\hline Neurofisiologia do exercício & & & & & 2 \\
\hline Neurofisiologia experimental & & & & 1 & 1 \\
\hline $\begin{array}{l}\text { Intervençốes terapêuticas no Transtorno de Déficit de Atenção com } \\
\text { Hiperatividade e epilepsia }\end{array}$ & & & & 1 & 1 \\
\hline Neurofisiologia do sono & & & & 1 & 2 \\
\hline Ensino de fisiologia & 1 & & & 1 & 1 \\
\hline Neurofisiologia da má nutrição & & 1 & & & \\
\hline Neurofisiologia da audição e reflexos audiomotores & & 1 & 1 & 1 & \\
\hline Neurofisiologia da medula espinhal & & & & 1 & \\
\hline Neurofisiologia do controle dos movimentos & & & & 1 & \\
\hline
\end{tabular}

1-(N) Norte; 2-(Ndt) Nordeste ; 3-(CO) Centro-Oeste; 4-(Sdt) Sudeste; 5-(S) Sul. 
Verificamos ainda, que 71,15\% ( $\mathrm{n}=37)$ dos GP em neurofisiologia estão vinculados a IES federais, o que pode ser observado na Figura 1A. A Figura 1B mostra que a prevalência dos GP em neurofisiologia encontra-se na região sudeste, $46,15 \%(\mathrm{n}=24)$, seguido da sul, $28,85 \%$ $(n=15)$.

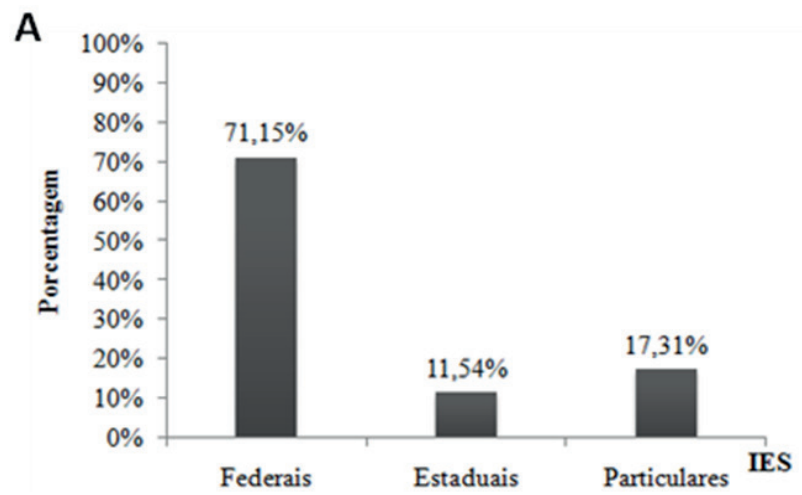

B

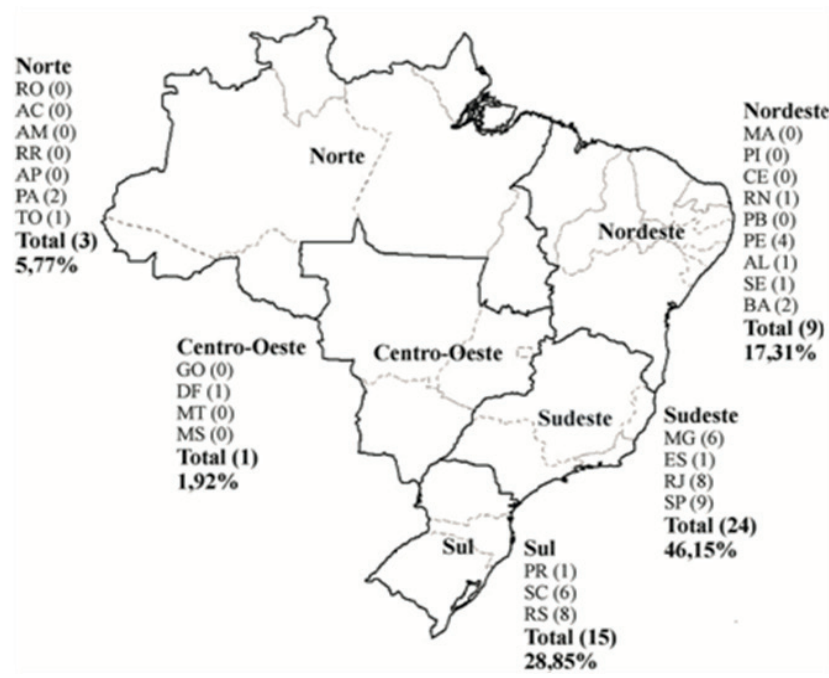

Figura 1. Distribuição dos Grupos de Pesquisa em Neurofisiologia de acordo com os tipos de Instituições de Ensino Superior (IES; A) e com as regiốes do Brasil (B).

Quase metade dos grupos de pesquisa incluídos nesta investigação iniciou suas atividades há menos de 5 anos, sendo que o ano de 2010 apresentou maior número de formações de GPs (Tabela 2).

Contabilizando os membros de todos os grupos, que incluem técnicos, estudantes e pesquisadores, temos 1326 participantes, dos quais $61,46 \%(n=815)$ são estudantes, 35,0\% $(n=464)$ são pesquisadores e 3,54\% $(\mathrm{n}=47)$ corpo técnico (Figura 2).
A análise do currículo de cada líder junto à Plataforma Lattes, revelou que todos os líderes possuem doutorado $(n=52)$ e a maioria pós-doutorado $(n=32)$. A produção bibliográfica e técnica, e o número de orientações concluídas no período de 2010 a 2013, respectivamente, apresentam prevalência na região sul, seguida da sudeste, conforme pode ser observado na Figura 3A. Este dado pode estar relacionado com a maior concentração de estudantes e pesquisadores na regiáo sul e de técnicos na região sudeste do país (Figura 3B).

Tabela 2

Número de Grupos de Pesquisa em Neurofisiologia formados por ano entre 1980 e 2012, no Brasil

\begin{tabular}{|c|c|}
\hline Ano de formaçáo & $\mathbf{N}^{\circ}$ de grupos criados \\
\hline 1980 & 1 \\
1981 & 1 \\
1989 & 1 \\
1990 & 3 \\
1995 & 2 \\
1996 & 4 \\
1999 & 1 \\
2000 & 4 \\
2004 & 1 \\
2005 & 1 \\
2006 & 2 \\
2007 & 3 \\
2008 & 4 \\
2009 & 3 \\
2010 & 8 \\
2011 & 2 \\
2012 & 3 \\
\hline
\end{tabular}

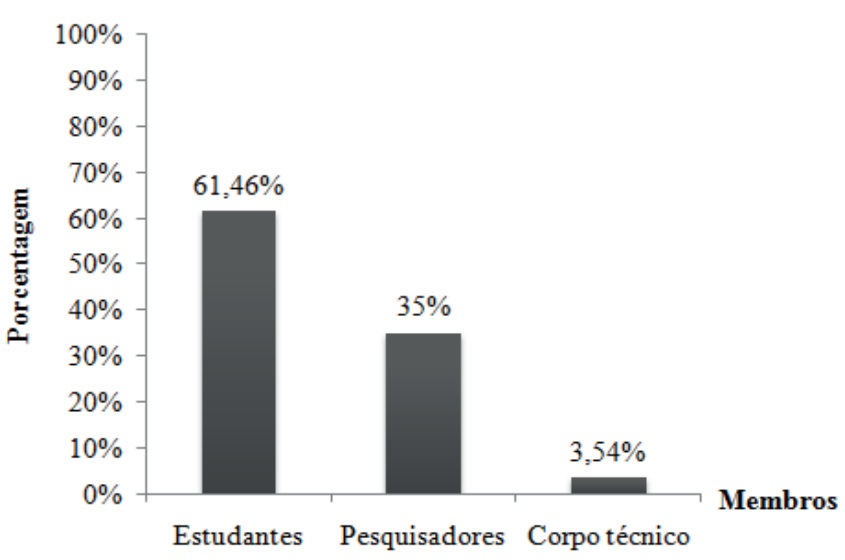

Figura 2. Distribuição dos Integrantes dos Grupos de Pesquisa em Neurofisiologia por categoria (estudantes, pesquisadores e técnicos). 
A Figura 3C apresenta a distribuição dos membros dos GP em neurofisiologia por habitante da região. Percebe-se que a regiáo sul apresenta 1 membro de GP em neurofisiologia para cada 59.403 habitantes, enquanto a região sudeste apresenta 170.240 habitantes/por membro.

\section{DISCUSSÃO}

Os resultados apresentados apontam que as linhas de pesquisa encontradas, embora apresentem diversidade, não estão distantes do tema central da pesquisa, que é a neurofisiologia, o que revela abrangência e interdisciplinaridade do tema, e que há maior variedade de linhas de pesquisa na regiáo sul do país.

Ainda sobre a distribuição dos GP, verificamos que $71,15 \%$ dos GP em neurofisiologia estâo vinculados a IES federais, o que pode ser justificado principalmente pelo compromisso que estas têm com o desenvolvimento do próprio Estado, com especial destaque para as políticas educacionais ${ }^{14}$, além de receberem apoio de importantes órgãos de fomento a pesquisa, como a Fapesp, no caso do estado de São Paulo. Assim, fica claro que, apesar das intensas transformaçóes que o sistema brasileiro de educação superior tem passado na última década, e do exponencial crescimento das instituiçóes acadêmicas, principalmente de caráter privado, a pesquisa continua sendo fomentada prioritariamente em Instituiçôes Federais de Ensino Superior (IFES) ${ }^{14}$.

Apesar de ocorrerem, ao longo das duas últimas décadas, movimentos de descentralização das atividades de fomento federal à ciência, tecnologia e inovação, com o estabelecimento de diversas parcerias entre instituições federais e estaduais ${ }^{15}$, verificamos que a prevalência dos GP em neurofisiologia encontra-se nas regiōes sudeste, $46,15 \%(n=24)$ e sul, 28,85\% ( $n=15)$. Este fato pode ser justificado pelo desenvolvimento econômico e educacional que esta região apresenta em relação às outras. Dados de 2010 do Instituto Brasileiro de Geografia e Estatística (IBGE) indicam que a região sudeste apresenta mais da metade $(55,4 \%)$ da participação no PIB (Produto Interno Bruto) do país ${ }^{16}$, além de maior número de IES e matrículas em cursos de graduação em relação às outras regióes do país ${ }^{17}$. Assim, existe na região sudeste, a concentração de financiamento e centros de excelência tanto no setor de pesquisa em saúde quanto na distribuição dos recursos humanos, revelando iniquidades regionais quando comparado com as regióes Norte, Nordeste e Centro-Oeste do Brasil ${ }^{18}$.

$\mathrm{O}$ fato de quase metade dos grupos de pesquisa incluídos nesta investigação terem iniciado suas atividades a menos de 5 anos, sendo que o ano de 2010 apresentou maior número de formaçóes de GPs, pode estar relacionado com a expansão no ensino superior no Brasil na última década ${ }^{19}$, à evolução da pesquisa científica no Brasil e a consolidação da política de pós-graduação implantada nos anos 60 nas principais IES ${ }^{20}$.

Considerando que as finalidades da educação superior não são simples nem unidimensionais, mas envolvem, ao contrário, um conjunto intencional e subjetivo que torna a formação profissional mais abrangente do que somente as açóes educativas encontradas numa estrutura curricular ${ }^{21}$, a formação acadêmica deve abranger outros ambientes. Assim, embora haja uma significante parcela de estudantes envolvidos quando comparado ao número de pesquisadores e corpo técnico, verifica-se que os estudantes representam apenas cerca de dois alunos por pesquisador.

Portanto, a participação em grupos de pesquisa privilegia a articulaçáo de conhecimentos e competências, e consiste em desenvolver relaçóes entre a aprendizagem cognitiva e experiencial, além do desenvolvimento de uma postura ética, crítica, de relacionamento pessoal e grupal e a construção de uma vivência acadêmica atuan$\mathrm{te}^{22}$. Assim, fica claro que a participação de alunos nos GP brasileiros precisa, ainda, ser fomentada.

Destaca-se também o número reduzido de corpo técnico observado, o que pode dificultar a manutenção dos laboratórios de pesquisa, importantes ferramentas para produção científica nesta área. Além de que o papel do técnico na formação dos GP reside na sua importância de fortalecer a articulação entre o ensino e o serviço, por meio da sua vivência e experiência profissional. Esta articulação é essencial aos GP visto que o conhecimento é culturalmente produzido, e o meio social é fundamental para esta construção, pois o conhecimento não emerge dos sujeitos, nem dos objetos, mas de uma relação dialética entre sujeito (conhecedor) e o objeto (conhecido) ${ }^{23}$. Dessa forma, os GP estruturam-se como um importante espaço para a inter-relação entre a teoria e a prática, favo- 

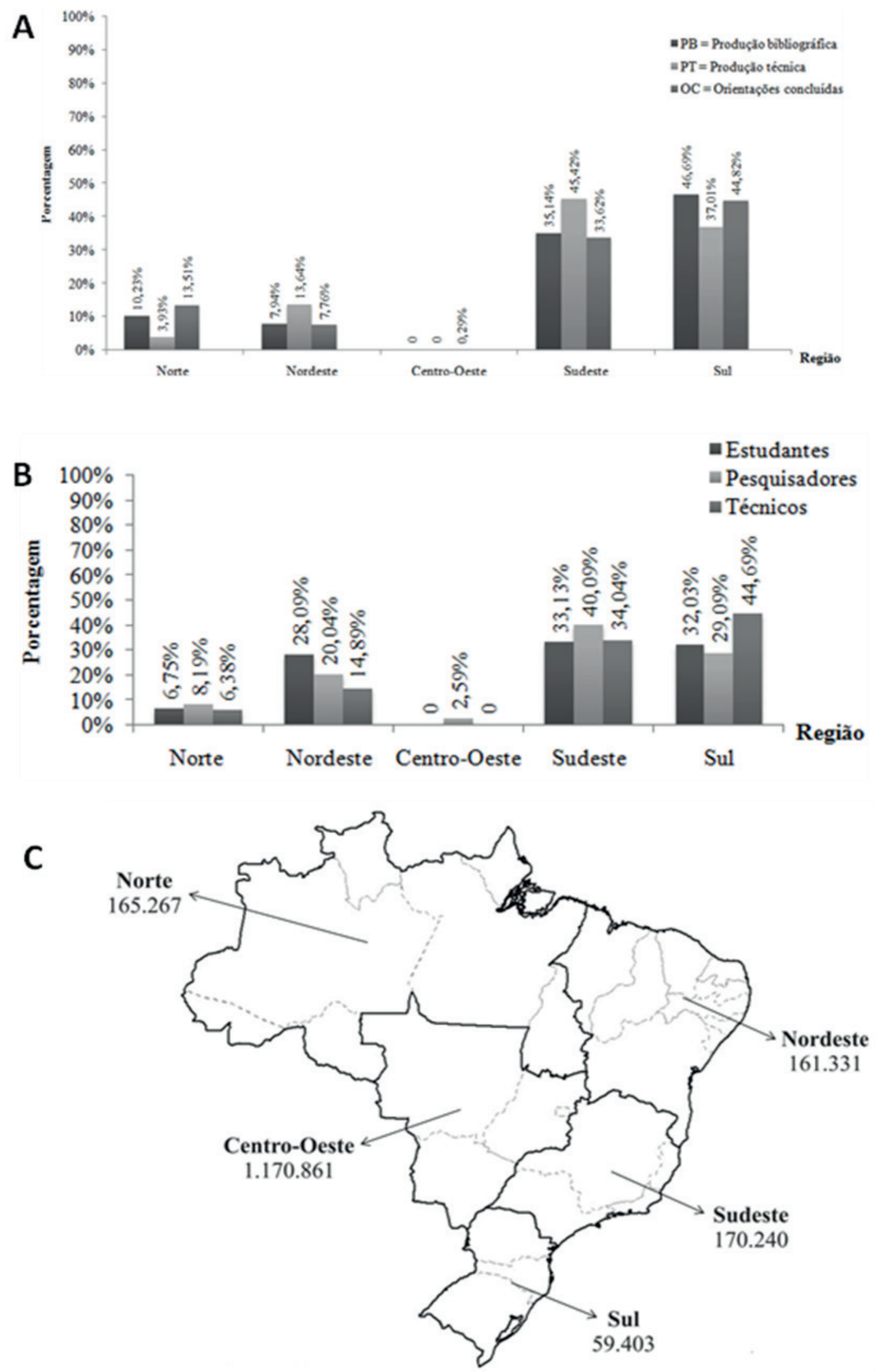

Figura 3. Distribuição regional da produção e orientações dos líderes dos GP em Neurofisiologia entre 2010 e 2013 (A), dos integrantes de diferentes categorias dos GP em Neurofisiologia (B) e do número de integrantes dos GP em Neurofisiologia/habitante (C). 
recendo o desenvolvimento e fortalecimento de uma visão sistêmica e abrangente, além de valorizar os diferentes modos de pensar e produzirir ${ }^{24}$.

A alta qualificação dos pesquisadores, demonstrada pela análise de seus currículos, é fator fundamental para o desenvolvimento científico do país. Ainda, a produção bibliográfica e técnica, e o número de orientações concluídas são importantes indicativos de produtividade científica e formação de recursos humanos, e, neste caso, concentram-se nas regióes sul e sudeste, respectivamente. Este dado pode estar relacionado com a maior concentração de estudantes e pesquisadores na região sul e de técnicos na região sudeste do país. Observa-se, desse modo, a concentração da produção científica no eixo Sul-Sudeste e urge a necessidade de criação de políticas de descentralização do conhecimento, além da distribuição igualitária de financiamentos de pesquisas, a fim de suprir as desigualdades entre as regióes e consequentes produçóes ${ }^{25}$.

\section{CONCLUSÃO}

Os resultados apresentados permitem conhecer e analisar as atividades dos GP em neurofisiologia existentes no Brasil, e dessa forma, traçar um perfil dos temas, quantidade de membros e prevalências regional e institucional, entre outros, possibilitando uma reflexão acerca desses dados.

Verificamos que, nos últimos anos houve um aumento no número de GP em neurofisiologia e a expansão da produção científica nesta área específica, porém a maioria dos GP, pesquisadores e estudantes concentram-se nas regiões Sul e Sudeste. Atualmente não se encontram muitos estudos que, como este, revelem a produção científica e atividades desenvolvidas por pesquisadores e GP de áreas específicas, assim há a necessidade de mais estudos deste tipo, para que sejam disponibilizadas informações que auxiliem na promoção da cultura científica.

\section{REFERÊNCIAS}

1.Schmidek WR, Cantos GA. Evolução do sistema nervoso, especializaçáo hemisférica e plasticidade cerebral: um caminho ainda a ser percorrido. Rev Pensam Biocen 2008;10:181-204.

2.Ribas GC. Consideraçôes sobre a evolução filogenética do sistema ner- voso, o comportamento e a emergência da consciência. Rev Bras Psiquiatr 2006;28:326-38.

http://dx.doi.org/10.1590/S1516-44462006000400015

3.Pizetta JR. O cérebro e a aprendizagem. (endereço na Internet). Maringá: Revista Científica Eficaz. (última atualização 01/2011; citado em 06/2013). Disponível em: http://www.faculdadeeficaz.com.br/revistacientificaeficaz/artigo/educacao/2011/ed_01/Jos\%C3\%A9-Pizetta.pdf.

4.Alvarenga SP. Contribuição da neurociência no processo ensino-aprendizagem em alunos com paralisia cerebral (Tese). Rio de Janeiro: AVM Faculdade Integrada, 2012, 39p.

5.História da Neurofisiologia no Brasil (endereço na Internet). São Paulo: Sociedade Brasileira de Neurociências e Comportamento. (última atualização 06/2013; citado em 06/2013). Disponível em: http://www.sbnec.org.br/site/ index.php?page=historia.

6.Sociedade Brasileira de Neurofisiologia Clínica (endereço na Internet). São Paulo: Sociedade Brasileira de Neurofisiologia Clínica. (última atualização 03/2014; citado em 03/2014). Disponível em: http://www.sbnc.org.br/.

7.Bacheschi LA, Guerreiro CAM. Situação das neurociências no Brasil: neurociências clínicas. Cienc Cult 2004;56:25.

8.Schveitzer MC, Backes VMS, Prado ML, Lino MM, Ferraz F. Grupos de pesquisa em educação em enfermagem: linhas de pesquisa e produção científica em três regiốes do Brasil. Rev Bras Enferm 2012;65:332-8.

http://dx.doi.org/10.1590/S0034-71672012000200020

9.Castrillón MC. Trends and Priorities in Nursing Research. Rev Latinoam Enferm 2004;12:853-8.

10.Grupos de Pesquisa: censos (endereço na Internet). Brasil: Ministério da Ciência e Tecnologia. (última atualização 06/2013; citado em 06/2013). Disponível em: http://dgp.cnpq.br/censos.

11.Grupos de Pesquisa em Ciência da Informação no Brasil (endereço na Internet). Campinas: Simpósio sobre o impacto das tecnologias na pesquisa e pós-graduação em Ciência da Informação/60 Reunião Anual da SBPC. (última atualização 07/2008; citado em 06/2013). Disponível em: http://www. sbpcnet.org.br/livro/60ra/textos/SI-MarisaBrascher.pdf.

12.Prado SD, Sayd JD. A pesquisa sobre envelhecimento humano no Brasil: grupos e linhas de pesquisa. Ciênc saúde coletiva 2004;9:57-68.

http://dx.doi.org/10.1590/S1413-81232004000100006

13.Grupos de Pesquisa (endereço na Internet). Brasil: Conselho Nacional de Desenvolvimento Científico e Tecnológico. (última atualização 06/2013; citado em 06/2013). Disponível em: http://memoria.cnpq.br/gpesq/apresentacao. htm\#p1.

14. Moraes M, Piccinini AM, Mello PB, Rocha VM. Panorama dos Cursos de Fisioterapia da Região Sul do Brasil. Interface 2011;8:57-72.

15.Botelho A, Almeida M. Desconstruindo a política científica no Brasil: evolução da descentralização da política de apoio à pesquisa e inovação. Soc estado. 2012;27:117-32.

http://dx.doi.org/10.1590/S0102-69922012000100008

16.Participação percentual das Grandes Regióes no Produto Interno Bruto 2002-2010 (endereço na Internet). Brasília: Instituto Brasileiro de Geografia e Estatística. (última atualização 11/2012; citado em 06/2013). Disponível em: http://saladeimprensa.ibge.gov.br/noticias?view=noticia\&id=1\&busca $=1 \& i \mathrm{dn}$ oticia $=2265$.

17.Censo da educação superior: 2011-Resumo Técnico (endereço na Internet). Brasília: Instituto Nacional de Estudos e Pesquisas Educacionais Anísio Teixeira. (última atualização 04/2013; citado em 06/2013). Disponível em: http://download.inep.gov.br/educacao_superior/censo_superior/resumo_tecnico/resumo_tecnico_censo_educacao_superior_2011.pdf.

18.Gomes DC, Backes VMS, Lino MM, Canever BP, Ferraz F, Schveitzer MC. Produção científica em Educação em Enfermagem: grupos de pesquisa Rio de 
Janeiro e Minas Gerais. Rev Gaúch Enferm 2011;32:330-7.

http://dx.doi.org/10.1590/S1983-14472011000200017

19.Giannetti E. A civilizaçáo brasileira/Idéias para quem decide. Rev Exame Ceo 2010;16-33.

20.UNICAMP. Desafios da pesquisa no Brasil: uma contribuição ao debate. São Paulo em Perspectiva 2002;16:15-23.

21.Peres CM, Andrade AS, Garcia SB. Atividades Extracurriculares: Multiplicidade e Diferenciação Necessárias ao Currículo. Rev Bras Educ Méd 2007;31:147-55.

http://dx.doi.org/10.1590/S0100-55022007000300002
22. Caires S, Almeida LS. Os estágios na formação dos estudantes do ensino superior: tópicos para um debate em aberto. Rev Port Educ 2000;13219-41.

23. Kincheloe JL. A formação do professor como compromisso político: mapeando o pós-moderno. Porto Alegre: Artes Médicas, 1997, 262p.

24.Backes VMS, Canever BP, Ferraz F, Lino MM, Prado ML, Reibnitz KS. Grupos de pesquisa de educação em enfermagem da Região Sul do Brasil. Rev Gaúcha Enferm 2009;30:249-56.

25.Santos A, Bastos LLAG, Aleixo AA, Paulo TRS, Mendes EL. Distribuição, evoluçấo e produção científica dos grupos de pesquisa em atividade física e saúde do Brasil. Rev Bras Ativ Fis e Saúde 2012;17:258-62. 